\title{
Factores asociados a la victimización en episodios de violencia escolar ${ }^{15}$
}

\author{
Daniel Fregoso Borrego \\ Magister en Desarrollo Regional \\ Centro de Investigación en Alimentación y Desarrollo, A.C. México \\ Correo electrónico: daniel_fregoso14@hotmail.com \\ Jose Angel Vera Noriega \\ Doctor en Psicología Social \\ Centro de Investigación en Alimentación y Desarrollo, A.C. México \\ Correo electrónico: avera@ciad.mx \\ Karen Guadalupe Duarte Tanori \\ Magister en Desarrollo Regional \\ Centro de Investigación en Alimentación y Desarrollo, A.C. México \\ Correo electrónico: kareng_18@hotmail.com
}

\section{Francisco Justiniano Velasco Arellanes \\ Doctor en Ciencia de la Conducta. Universidad de Sonora, México Correo electrónico: francisco.velasco@unison.mx}

Recibido: 26/09/2019

Evaluado: $13 / 01 / 2020$

Aceptado: 20/01/2020

\section{Resumen}

La violencia escolar ha llamado la atención por las consecuencias en la salud y el rendimiento académico de la juventud. Los factores familiares se han asociado con la violencia escolar. Objetivo: Modelar la relación de algunas variables implicadas en el factor escolar y familiar con víctimas en el fenómeno de la violencia escolar. Método: Participó una muestra de 669 díadas estudiante-padre. Se realizaron tres modelos, uno para el factor escolar, otro para el factor familiar y un tercero para establecer vínculos entre ambos factores. Resultados: Los modelos presentaron índices de bondad de ajuste adecuados. El rol de espectador y empatía hacia la víctima están relacionados. La comunicación que establecen los padres con los estudiantes se vincula con el control y promoción de habilidades sociales. Conclusión: Los modelos son preliminares y se requieren considerar variables empíricas que cotejen estas y más relaciones entre estos dos factores.

Palabras clave

Violencia escolar, salud, familia, escuela.

15 Para citar este artículo: Fregoso, D., Vera, J. A., Duarte, K. G., y Velasco, F. J. (2021). Factores asociados a la victimización en episodios de violencia escolar. Informes Psicológicos, 21(1), 231-245 http://dx.doi.org/10.18566/infpsic.v21n1a15 


\title{
Factors associated with victimization in episodes of school violence
}

\begin{abstract}
School violence has drawn attention due to the consequences on the health and academic performance of youth. Family factors have been associated with school violence. Objective: To model the relationship of some variables involved in the school and family factor with victims in the phenomenon of school violence. Method: A sample of 669 student-parent dyads participated. Three models were made, one for the school factor, another for the family factor and a third to establish links between both factors. Results: The models presented adequate goodness of fit indices. The role of spectator and empathy towards the victim are related. The communication that parents establish with students is linked to the control and promotion of social skills. Conclusion: The models are preliminary and it is necessary to consider empirical variables that compare these and more relationships between these two factors.
\end{abstract}

Keywords

School violence, health, family, school.

\section{Fatores associados à vitimização em episódios de violência escolar}

\section{Resumo}

A violência escolar tem chamado a atenção para as consequências na saúde e no desempenho acadêmico dos jovens. Os fatores familiares têm sido associados à violência escolar. Objetivo: Modelar a relação de algumas variáveis envolvidas no fator escola e família com as vítimas do fenômeno da violência escolar. Método: Participou uma amostra de 669 díades aluno-pais. Foram feitos três modelos, um para o fator escola, outro para o fator família e um terceiro para estabelecer ligações entre os dois fatores. Resultados: 0s modelos apresentaram índices de bondade de adequação. 0 papel de espectador e a empatia para com a vítima estão relacionados. A comunicação estabelecida pelos pais com os alunos está ligada ao controle e promoção de habilidades sociais. Conclusão: Os modelos são preliminares e é necessário considerar variáveis empíricas que verifiquem essas e mais relações entre esses dois fatores. 


\section{ntroducción}

La violencia escolar se ha abordado a partir de dos perspectivas: 1) desde la visión del delito, y 2) desde el ámbito escolar. Se caracteriza por conductas disruptivas como el asalto, el robo, la agresión, la indisciplina, el vandalismo y el daño de instalaciones (Muñoz, 2008). De las distintas formas como puede presentarse la violencia escolar, la que más atención ha recibido en los últimos años, por su frecuencia y graves consecuencias, es la agresión entre pares (Valdés, Yáñez \& Carlos, 2013). La agresión entre pares es un fenómeno social que consiste en actos ofensivos intencionales que dañan física, psicológica y/o emocionalmente a algún compañero (Parke \& Slaby, 1983).

Dentro de un evento de agresión entre pares, coexisten tres actores principales: el agresor, la víctima y los espectadores (Coloroso, 2004). Específicamente, los agresores se caracterizan por ser impulsivos, tener baja tolerancia a la frustración, tener dificultad para cumplir normas y poseer una actitud positiva hacia la violencia (Díaz-Aguado, 2006; Olweus, 1993). Las consecuencias para los agresores implican el desarrollo de conductas violentas como forma de relacionarse y el incremento de riesgo de convertirse en un delincuente en su adultez (Farrington, 1993; Sharp, Thompson \& Arora, 2000).

Por su parte, las víctimas suelen padecer problemas como baja autoestima, actitudes pasivas, trastornos emocionales, problemas psicosomáticos, depresión y ansiedad (Cepeda, Pacheco, García \& Piraquive, 2008; Da Silva, Kodato \& Xavier, 2013; Olweus, 1993). Las víctimas tienen consecuencias más graves en su psique como sufrimiento por soledad, ideación e intención suicida, pérdida de interés por los estudios; lo que puede acontecer, a su vez, en bajo rendimiento académico, abandono escolar o consumación del suicidio (Kochenderfer \& Ladd, 1996).

Cualquiera que sea el tipo de agresión, tanto para víctimas como agresores, se presenta propensión a la ideación suicida, ansiedad, angustia, comportamientos antisociales, depresión, bajo rendimiento académico o deserción escolar (Leff, Power \& Goldstein, 2004; Rigby, 2000).

La agresión entre pares crea un sentimiento de vulnerabilidad general dentro de la escuela, afecta la calidad de las relaciones interpersonales y se presenta como un factor de riesgo escolar (Blaya, Debarbieux, Rey-Alamillo \& Ortega-Ruiz, 2006). Institucionalmente, la agresión entre pares daña el proceso de aprendizaje $\mathrm{y}$, por consiguiente, la calidad educativa (López, Bilbao \& Rodríguez, 2012).

El estudio de las víctimas es central para comprender los efectos de la violencia que se ejerce en las escuelas. Se ha encontrado que las respuestas de la víctima hacia la violencia implican un historial duradero de conductas mal adaptadas para enfrentarse con compromiso y esfuerzo a las adversidades como las situaciones de estrés; finalmente, cuando no se cuenta con habilidades de afrontamiento, las víctimas tienden a caer en depresión (Troop-Gordon, Rudolph, Sugimura \& Little, 2014). 
Se ha encontrado que la vulnerabilidad o el afrontamiento que puedan tener los jóvenes ante la situación de violencia está relacionado con el clima, las actitudes y las relaciones familiares (Hlavaty \& Haselschwerdt, 2019; López \& Ovejero, 2015; Martínez-Ferrer, Murgui-Pérez, Musitu-Ochoa \& Monreal-Gimeno, 2008; Moral \& Ovejero, 2014). En esta línea de investigación Hlavaty y Haselschwerdt (2019) realizaron un estudio para evaluar si la coercitividad, el control sobre el ejercicio de la violencia física y la violencia doméstica se asocia con las relaciones entre pares como la victimización, la perpetración de violencia y la calidad de la amistad.

Los investigadores encontraron que en el grupo control ( $n=48$ ), los cuales no habían sufrido violencia doméstica, pero sí se ejercía en ellos coerción y control sobre la violencia (que se corrigieran los actos de violencia), se perfilaron en el rol de víctima y contaron con una mejor calidad de amistad. Por otra parte, el grupo que fue expuesto a violencia doméstica $(n=96)$ presentó una relación con sus pares de perpetración de acoso (rol de victimarios o agresores). Estos resultados sugieren que los jóvenes que no sufren violencia doméstica, y se les controla para no ejercerla, presentan una buena amistad con sus pares, pero pueden ser víctimas de acoso en el contexto escolar. Los que son expuestos a violencia doméstica son proclives a ser los perpetradores de violencia.

Por su parte, Moral y Ovejero (2014) realizaron un estudio sobre la relación entre el clima social familiar, en cuanto a la comunicación paterno-filial, empleo del castigo físico y control psicológico para disciplinar, y su relación con el comportamiento de los hijos en sociedad. Específicamente se buscó evaluar la relación del clima social familiar con el acoso en el contexto escolar. Se encontró que los adolescentes con actitudes favorables ante el ejercicio de la violencia entre pares perciben a su hogar con clima familiar más conflictivo, con más utilización de castigos corporales y violencia psicológica por parte de sus padres. Así mismo, presentan más dificultades de comunicación y menor comprensión y apoyo psicosocial con sus pares.

Así pues, las referencias anteriores indican que el ambiente, clima o contexto familiar influye en la relación entre pares $y$, en específico, en la violencia entre pares. Sin embargo, los estudios anteriores derivan sus resultados de datos pertenecientes sólo al estudiante, por lo que resulta importante obtener datos de la diada estudiante-padre, madre y/o tutores. De esta manera, el objetivo del presente estudio es modelar mediante ecuaciones estructurales la relación entre el factor familiar y el factor personal del estudiante con el rol víctima.

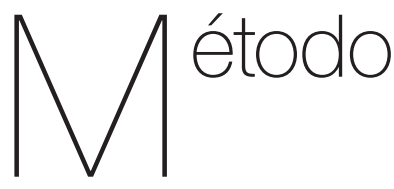

El presente trabajo es cuantitativo, con un diseño de tipo transversal, de carácter analítico y no experimental por cuotas establecidas por municipio y escuela; en cada región se eligieron aleatoriamente el número de escuelas según la población estudiantil en el municipio y después en 
cada escuela se eligió un primer, segundo y tercer grado de secundaria.

\section{Participantes}

En el estudio se consideró una muestra total de 669 estudiantes de secundaria (282 hombres y 387 mujeres) con sus padres, madres y/o tutores, los cuales fueron elegidos por cuotas en 61 escuelas de 17 municipios del Estado de Sonora, México. Del total de 2,354 estudiantes que participaron en una muestra representativa del estado de Sonora para estudiar clima y convivencia escolar, solo 669 regresaron con los instrumentos destinados a evaluar la percepción de alguno de los padres o tutores (madre, padre o tutores), siendo la tasa de no respuesta del $71.5 \%$. Así pues, solo las 669 diadas estudiantes-padres, madre y/o tutores que firmaron el consentimiento informado y completaron la medida, fueron considerados para este estudio. Por lo anterior, la muestra se considera no aleatoria y por conveniencia.

\section{Instrumentos}

Se aplicaron siete escalas que han sido validadas para medir aspectos de violencia escolar en población mexicana, las cuales son: escala agresor y víctima, escala de espectadores y escala de empatía (factor personal del estudiante), participación de los padres en la educación de sus hijos, control y promoción de habilidades sociales (factor familiar). Además, se formaron las variables Sociocultura y Disfunción, las cuales están integradas por reactivos que miden situaciones familiares del orden cultural y de convivencia.
A continuación, se presenta cada uno de los instrumentos señalando sus características, tipo, origen y su validez de constructo en el estado de Sonora. Aunque los instrumentos son validados para el estado de Sonora, su validez aún es de indagarse para poblaciones más amplias y externas.

\section{Factor personal (estudiantes). Escala de agresores y víctimas.}

Esta escala es una adecuación a partir de la creada por Del Rey y Ortega (2007) que contaba con 80 ítems de tipo Likert para evaluar el comportamiento agresivo hacia compañeros. Sus dimensiones son agresiones de índole física, social, psicológica, sexual y cyberbullying. La adecuación fue realizada por González, Peña y Vera (2017) la cual tuvo como propósito identificar las conductas de agresión emitidas, pero también recibidas en relación con las dimensiones antes mencionadas.

Se obtuvieron 12 ítems tipo Likert (1 = Nunca a 5 = Siempre) que describen las agresiones emitidas y otros 12 ítems que explican las agresiones recibidas; a diferencia de Del Rey y Ortega (2007), las dimensiones fueron dos: violencia verbal/relacional (siete ítems) y violencia física/social (cinco ítems). La validación se realizó a través de Análisis Factorial Exploratorio (AFE), análisis de Rasch y Análisis Factorial Confirmatorio (AFC). Los valores de validación para cada análisis son los siguientes: ajuste externo e interno de .54 a 1.35 y .75 a 1.24 , respectivamente; KMO de .90; varianza total explicada de 39\%; alfa de Cronbach de .85; CFI de .90; RMSEA de .08; AGFI 
de .90; SRMR de .05 y GFI de .93. Las cargas factoriales se encontraron de .50 a .78 en las dos dimensiones. Todos fueron indicadores aceptables por lo cual se puede decir que la escala es válida (González et al., 2017). Para propósitos de la investigación, sólo se utilizó la escala de víctimas.

\section{Escala de espectadores.}

Desarrollada por Belacchi y Fanina (2010) para evaluar los diferentes tipos de espectadores que se asumen en un episodio de agresión en la escuela. González et al. (2017) adaptaron la escala a la población mexicana, específicamente, en Sonora. Esta versión se conformó por 13 ítems. Los reactivos se responden en una escala Likert de cinco opciones (1 = Nunca a 5 = Siempre) la cual permite evaluar tres tipos de observador: 1) reforzador, 2) defensor y 3) evasivo. Cabe señalar que el instrumento de Belacchi y Fanina (2010) permite evaluar siete roles (agresor, víctima, defensor, reforzador, mediador, evasivo y consolador); sin embargo, algunos fueron eliminados debido a que la metodología que se requería para evaluar este rol era diferente en relación a los otros roles.

Los indicadores de validez fueron los siguientes: CFI de .96; GFI de .96; RMSEA de .05; SRMR de .04; AGFI de .95; alfa de Cronbach de .82; cargas factoriales no menores a .60 y no mayores a .83; ajustes internos y externos adecuados pues van de .83 a 1.15 y de .68 a 1.26 respectivamente, $\mathrm{KMO}$ de .89 y una varianza total explicada de 56.98\% (González et al., 2017).
Escala de Empatía.

Bautista, Vera, Tánori y Valdés (2016) evaluaron la pertinencia de integrar y validar un instrumento de 20 reactivos para medir empatía en población de Sonora. Ésta se integró a partir de 15 reactivos del cuestionario de empatía de Toronto (Spreng, McKinnon, Mar \& Leviene, 2009) el cual mide la dimensión emocional y de cinco reactivos tomados de Davis (1980) que miden la dimensión cognitiva o bien toma de perspectiva. El instrumento fue ajustado previamente en estudiantes de secundaria pública del municipio de Hermosillo, con el fin de mejorar la redacción de los reactivos (Bautista et al., 2016). Las preguntas se responden con escala de tipo Likert con cinco niveles de respuesta equilibrada (1 = Nunca a $5=$ Siempre). Los valores de validación obtenidos fueron los siguientes: CMIN de 3.74; CFI de .99; RMSEA de .30; SRMR de .16 y alfa de Cronbach de .85 .

Factor familiar. Participación de los padres en la educación de sus hijos.

Basado en Valdés, Carlos y Arreola (2013) está integrado por ocho reactivos tipo Likert con cinco puntos ( $1=$ Nunca a 5 = Siempre). El instrumento se aplica con el fin de saber qué tan informados están los padres sobre los docentes, el funcionamiento de la escuela, sobre la relación de sus hijos con sus compañeros y docentes de la escuela. Esta medida fue validada con una muestra representativa en Sonora por Duarte y Fregoso (2016) a partir del AFE, el cual arrojó dos dimensiones: padres en comunicación con la escuela y padres en comunicación con sus hijos con alfa de Cronbach de .84 y .80; 
KMO de 80 y varianza explicada de $76 \%$, seguido del análisis de Rasch donde se calibraron todos los reactivos $y$, por último, se consolidó con el AFC con cargas factoriales óptimas e índices de bondad de ajuste buenos con CFI de .96; RMSEA de .07 y SRMR de .04 .

\section{Escala de control y promoción de las habilidades sociales.}

Esta escala está formada por ocho reactivos. Mide la capacidad y eficacia percibida de los padres, con respecto a la gestión del control y promoción de las habilidades sociales de sus hijos. Está basada en la escala que propuso Bandura (2003) para medir autoeficacia. La validación en Sonora con una muestra representativa de Vera, Fregoso y Duarte (2017) mostró la unidimensionalidad de la escala con alfa de .90; KMO de .91 y varianza acumulada de 59\%; ajuste al modelo de Rasch sin obstaculizaciones e índices de bondad de ajuste buenos con CFI de .95; RMSEA de .09 y SRMR de .03. El formato de respuesta es tipo Likert de cinco puntos $(1=$ Nunca a 5 = Siempre).

\section{Sociocultura.}

Es una sumatoria compuesta de reactivos que permiten conocer las veces que, durante el año, los padres, madres, tutores y/o tutoras auspician el desarrollo de sus hijos mediante asistencias al cine, teatro, ferias o exposiciones culturales, si se practica algún deporte, leen libros, o bien, si tienen computadora y/o internet en casa, así como el nivel educativo de los padres (Armendáriz et al., 2015; Aurelia, 2005; Chartier, Hesselbrock \& Hesselbrock, 2010).

\section{Disfunción familiar}

Los reactivos que comprenden esta variable se encuentran en el orden de si alguno de los padres se encuentra preso en un centro de rehabilitación social, consumen alcohol o alguna droga, si ha recibido tratamiento psiquiátrico o psicológico y si los hijos han sido reportados por problemas de conducta en la escuela (Acero, Escobar, Franklin \& Castellanos, 2007; Omar, Urteaga, Uribe \& Soares, 2010; Pichardo, Fernández \& Amezcua, 2002; Sanabría \& Uribe, 2010).

Para obtener las dimensiones de cada escala se utilizó la media aritmética sumando los reactivos identificados en cada sub-escala, y se dividió entre el número de reactivos que las formaban.

\section{Procedimiento}

Los instrumentos utilizados para la obtención de datos sobre el rol de victimización y de empatía fueron aplicados a los estudiantes en el salón de clases. Para obtener la información de familia, los instrumentos fueron enviados, en sobre cerrado, a los hogares a través de los estudiantes quienes llevaron las respuestas el día siguiente.

También se les hizo entrega de un consentimiento informado el cual fue firmado por los estudiantes y sus padres, madres y/o tutores involucrados. En este consentimiento se les informó el propósito del estudio y las intenciones que se tuvieron con respecto a la información que brindaron al contestar los instrumentos. Es importante mencionar que el trabajo de campo fue 
llevado a cabo en concordancia con los artículos 118 y 122 del Código Ético del Psicólogo, de la Sociedad Mexicana de Psicología (2007), los cuales hacen referencia al proceso de consentimiento informado de los estudiantes participantes.

La investigación también fue basada en el artículo 136 del mismo código ético, que habla acerca de la confidencialidad de la información recabada en estudios de investigación, ya que se instruyó tanto a directivos de las escuelas, docentes y estudiantes acerca de los usos de los datos recopilados, así como el respeto al anonimato de los mismos.

\section{Análisis de datos}

El criterio para considerar que la aplicación de instrumentos era válida para participar en el estudio fue que el participante respondiera más del 90\% de los reactivos, eliminando a los participantes que respondían en un porcentaje menor. En el caso de aquellos participantes que respondieron más del $90 \%$ del instrumento, y que contaban con datos perdidos, estos se sustituyeron por la moda. Una vez identificadas las diadas estudiante-padre, y dada la validación previa de todos los instrumentos, se procedió a realizar ecuaciones estructurales. Se consideraron los factores que conforman a cada instrumento y se obtuvieron los modelos por separado, y al final integrando todas las variables.

En cuanto a la normalidad multivariante, se obtuvo a través de AMOS y observamos sólo el contraste para la curtosis multivariante y no incluye la asimetría, cuya estimación fue de 52.11 y valor experimental (critical ratio) de 20.49. Por lo anterior, podemos concluir que conjuntamente las variables presentan una curtosis significativamente distinta de la de una normal multivariante.

Para llevar a cabo los análisis de datos se empleó el programa estadístico Stadistical Package for the Social Sciences (SPSS) versión 25 y el software de modelado de ecuaciones estructurales AMOS versión 23. Para ajustar los modelos y sus parámetros, se utilizó el estimador Máxima verosimilitud. Los indicadores a visualizar en las ecuaciones estructurales son el CMIN/DF; GFI; CFI; RMSEA; SRMR; y AIC, siendo los valores mínimos aceptables de 3 o menor, $\geq$ a $.9, \geq$ a .9 , s a $.05, \leq$ a .05 respectivamente, según Hair, Anderson, Tatham y Black (2010).

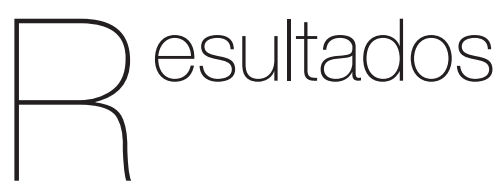

Se llevaron a cabo tres modelos en los cuales se involucraron las variables de padres, estudiantes y victimización. El primero se compuso únicamente por variables de los estudiantes con la victimización, el segundo por las variables de padres con victimización, y el tercer modelo trató de involucrar todas las variables antes mencionadas. En la Tabla 1 se muestran los valores obtenidos con sus índices de bondad de ajuste. 
Tabla 1

Muestra los valores obtenidos de los índices de bondad de ajuste para cada uno de los modelos.

\begin{tabular}{lcccccc}
\hline \multicolumn{1}{c}{ Modelo } & CMIN/DF & GFI & CFI & RMSEA & SRMR & AIC \\
\hline Víctima-estudiante & 2.2 & .9 & .92 & $.04(\mathrm{LO}=.03, \mathrm{HI}=.04)$ & .04 & 1396.84 \\
\hline Víctima-padres & 2.12 & .92 & .93 & $.04(\mathrm{LO}=.03, \mathrm{HI}=.04)$ & .03 & 1044.75 \\
\hline Modelo completo & 2.85 & .97 & .95 & $.05(\mathrm{LO}=.04, \mathrm{HI}=.06)$ & .04 & 186.62 \\
\hline
\end{tabular}

El modelo Víctima-escuela, en el cual se analiza el factor escuela implicado en la situación de victimización, resultó con medidas adecuadas, indicando todas ellas que el modelo es aceptable. Las cargas para cada uno de los factores integrantes del modelo fueron consideradas buenas (véase Tabla 2). Los valores obtenidos de las cargas factoriales se consideran aceptables según lo planteado por Hair et al. (2010). Todos estos valores en su conjunto ofrecen aceptabilidad del modelo.

Tabla 2

Muestra los valores de covarianza para cada variable para el modelo víctima-escuela, así como las cargas factoriales mínimas y máximas.

\begin{tabular}{|c|c|c|c|c|c|c|c|c|c|}
\hline \multirow{2}{*}{ Variable } & \multicolumn{7}{|c|}{ Covarianza } & \multicolumn{2}{|c|}{ Carga factorial } \\
\hline & VVVyR & VVFyS & Ref & Def & Eva & $\mathrm{EA}$ & EC & - & + \\
\hline VVVyR & - & & & & & & & .45 & .73 \\
\hline VVFyS & .83 & - & & & & & & .67 & .81 \\
\hline Reforzador & .29 & .27 & - & & & & & .64 & .73 \\
\hline Defensor & .12 & .03 & .17 & - & & & & .68 & .77 \\
\hline Evasivo & .09 & .05 & -.04 & .40 & - & & & .62 & .84 \\
\hline EA & -.04 & -.09 & -.10 & .34 & .25 & - & & .47 & .74 \\
\hline EC & -.10 & -.14 & -.12 & .30 & .24 & .88 & - & .51 & .77 \\
\hline
\end{tabular}

Nota: Víctima de violencia verbal y relacional (VVVyR); Víctima de violencia física y social (VVFyS); Reforzador (Ref); Defensor (Def); Eva (Evasivo); Empatía afectiva (EA); Empatía cognitiva (EC).

Bajo el modelo Victima-padres, se analizó el factor familiar ante la situación de violencia. Resultó con medidas adecuadas (véase Tabla 1) indicando todas ellas que el modelo es aceptable. Las cargas factoriales que se obtuvieron en el modelo fueron buenas (véase tabla 3). Los valores de $r$ fueron aceptables a partir de encontrarse iguales o mayores a .30 (Hair et al., 2010). Todos estos valores en su conjunto ofrecen aceptabilidad al modelo. 
Tabla 3

Muestra los valores de covarianza para cada variable para el modelo víctima-padres, así como las cargas factoriales mínimas y máximas.

\begin{tabular}{|c|c|c|c|c|c|c|c|c|c|}
\hline \multirow{2}{*}{ Variable } & \multicolumn{7}{|l|}{$\begin{array}{l}\text { Cova- } \\
\text { rianza }\end{array}$} & \multicolumn{2}{|c|}{ Carga factorial } \\
\hline & VVVyR & VVFyS & CP HS & CP-E & $\mathrm{CP}-\mathrm{H}$ & Sociocultura & Disfunc. & - & + \\
\hline VVVyR & - & & & & & & & .53 & .72 \\
\hline VVFyS & .83 & - & & & & & & .67 & .81 \\
\hline CP HS & -.08 & .27 & - & & & & & .60 & .79 \\
\hline CP-E & .02 & .03 & .17 & - & & & & .76 & .85 \\
\hline $\mathrm{CP}-\mathrm{H}$ & .00 & .05 & -.04 & .40 & - & & & .57 & .74 \\
\hline Sociocultura & -.04 & -.09 & -.10 & .34 & .25 & - & & .40 & .49 \\
\hline Disfunción & -.10 & -.14 & -.12 & .30 & .24 & .88 & - & .20 & .80 \\
\hline
\end{tabular}

Nota: Víctima de violencia verbal y relacional (VVVyR); Víctima de violencia física y social (VVFyS); Control y promoción de las habilidades sociales (CPHS); Comunicación padres-escuela (CP-E); Comunicación padres-hijos (CP-H).

Después se presenta el Modelo completo, el cual integra en un solo modelo a los modelos 1 y 2 (Víctima-escuela y Víctima-padres). Las cargas para cada uno de los factores del modelo completo fueron Víctima $(r=.91)$, Observadores $(r=$ .76), Empatía $(r=.85)$ y Comunicación ( $r=$ .93) (Véase Tabla 4).

Tabla 4

Muestra los valores de covarianza para cada variable para el modelo completo, así como las cargas factoriales mínimas y máximas.

\begin{tabular}{|c|c|c|c|c|c|c|c|c|c|}
\hline \multirow[b]{2}{*}{ Variable } & \multicolumn{7}{|c|}{ Covarianza } & \multicolumn{2}{|c|}{ Carga factorial } \\
\hline & Víctima & Obs & Empatía & Com & CPHS & Sociocultura & $\begin{array}{l}\text { Disfun- } \\
\text { ción }\end{array}$ & - & + \\
\hline Víctima & - & & & & & & & .65 & .91 \\
\hline Obs & .14 & - & & & & & & .12 & .76 \\
\hline Empatía & -.05 & .42 & - & & & & & .81 & .85 \\
\hline Com & -.02 & .03 & .11 & - & & & & .37 & .93 \\
\hline CPHS & -.08 & .05 & .09 & .47 & - & & & - & - \\
\hline Sociocultura & -.03 & .01 & .05 & .13 & .10 & - & & - & - \\
\hline Disfunción & .04 & .08 & -.07 & -.01 & -.06 & .14 & - & - & - \\
\hline
\end{tabular}

Nota: Observadores (Obs); Comunicación (Com); Control y promoción de las habilidades sociales (CPHS)

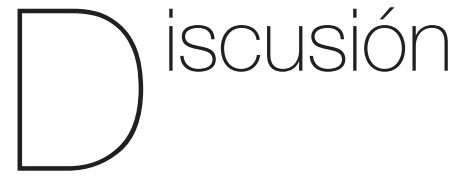

La investigación psicosocial de la violencia entre pares ha ofrecido evidencia de que factores familiares aportan a su explicación y cómo en ella se ejerce la paternidad para facilitar o interferir con actitudes y prácticas de la violencia. En el presente estudio se exploró mediante modelación con ecuaciones estructurales la relación entre el factor familiar 
(participación de los padres en la educación de sus hijos, control y promoción de habilidades sociales, promoción cultural y la disfunción en el hogar) y el factor escolar donde la violencia es ejercida (victimario, espectador, víctima) así como factores personales (empatía afectiva y cognitiva).

Los resultados del estudio indicaron, estadísticamente, que los factores familiar y escolar están implicados en el ejercicio de la violencia escolar. Aunque los tres modelos presentan índices de bondad de ajuste adecuados, pueden haber limitaciones empíricas para explicar los resultados, ya que es probable que la muestra de 669 diadas consideradas en este estudio pertenezcan a una población de padres de familia que tienen mayor interés por el desarrollo de sus hijos y que, si bien pueden tener condicionantes para el desarrollo y emergencia de la violencia escolar, estos pudiesen representar a la población de jóvenes menos vulnerables, pues se sabe que los padres que se involucran más con la formación de sus hijos y asisten a reuniones de trabajo en las escuelas, generalmente son padres de niños con mejores rendimientos académicos y con menos problemas de conducta (Betancourt \& Londoño, 2017; Solís \& Aguiar, 2017).

Así también, es importante considerar, como otra limitante, que el factor familiar fue evaluado desde la percepción que tienen los padres del hogar o la familia y no es la percepción de los estudiantes sobre la convivencia familiar; $y$, viceversa, el factor escolar fue evaluado desde la percepción de los estudiantes y no se cotejó con la percepción de los padres hacia la escuela. Bajo este contexto metodológico, podría ocurrir sesgo en términos de deseabilidad en el sentido de que ambos enfoques (percepción de estudiantes vs padres) no coinciden plenamente.

No obstante, a pesar de dichas limitantes iniciales, el modelo final presentó valores adecuados de ajuste mostrando una buena representación de la situación de victimización y de cómo los factores escolares y familiares interactúan. Principalmente la relación estrecha que se encuentra entre los tipos de violencia ejercida en las víctimas se presenta como consistente. Los modelos reflejan también, de manera congruente con la literatura, que los factores que protegen a la víctima de violencia son aquellos estudiantes que asumen el rol de espectador y la empatía que estos manifiestan con la víctima (covarianza, $r=.42$ ), así como con la calidad de la comunicación que tienen los padres con sus hijos en el hogar en relación con la socialización y búsqueda de solución de problemas de los hijos en la escuela (covarianza, $r=$ .47). Estos resultados respaldan lo que ya han encontrado investigadores como Hlavaty y Haselschwerdt (2019), Martínez-Ferrer et al. (2008) y Moral y Ovejero (2014) en términos de que la promoción de habilidades sociales en el hogar y el control de la violencia doméstica explican la relación entre pares en el contexto del acoso escolar.

De esta forma, el tercer modelo es el que aporta más robustez estadística. Esto podría deberse a que en este modelo se han considerado más variables contextuales en relación a la victimización y no solamente el reporte verbal de la víctima que está sujeta a violencia (ver Tabla 1). 


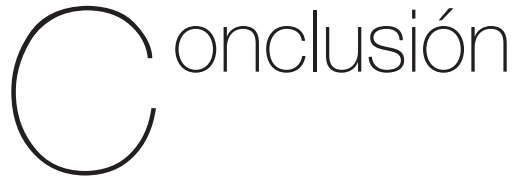

El estudio que se realizó es preliminar, es necesario considerar más variables empíricas que cotejen las relaciones entre los factores familiar y escolar. Es pertinente realizar estudios en los que se identifique y entreviste a los padres que tienen hijos con roles de víctima o victimarios para compararlos con los que no se comportan con ninguno de estos roles. Por otro lado, basados en la evidencia existente sobre el perfil de la víctima y los datos de comunicación de los padres con el estudiante y la escuela, el estudio podría indicar resultados congruentes a los de Cepeda et al. (2008), Da Silva et al. (2013) y Olweus (1993). Estos autores refieren una correspondencia entre la relación padre-hijo respecto al contexto escolar, la violencia escolar y las variables como la comunicación con los hijos, la vinculación con la escuela y la promoción de actividades sociales. Finalmente, bajo este contexto, es importante investigar cómo estas variables regulan la relación víctima-agresor, tal como lo sugieren Martínez-Ferrer et al. (2008) y Moral y Ovejero (2014). Así también, es preciso enfatizar que la violencia o coerción doméstica como modelo de convivencia entre los padres puede ser un condicionante para que los hijos sean proclives a ser víctimas en el ámbito escolar (Hlavaty \& Haselschwerdt, 2019). Lo anterior refleja la importancia de estudiar variables familiares adversas como la violencia o el uso de drogas entre sus integrantes para comprender la violencia que ocurre en el ámbito escolar.

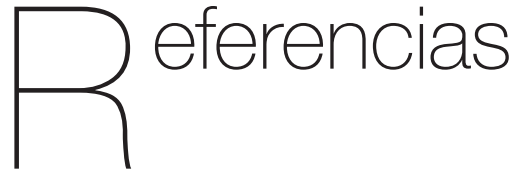

Acero, A., Escobar, Franklin \& Castellanos, G. (2007). Factores de riesgo para violencia y homicidio juvenil. Revista Colombiana de Psiquiatría, 36(1), 78-97.

Armendáriz, N., Almanza, J., Alonso, M., Oliva, N., Alonso, M. \& López, M. (2015). La historia familiar y la conducta de consumo de alcohol como factor sociocultural en el adolescente. Perspectiva de enfermería. Aquichan, 15(2), 219-227. doi: 10.5294/ aqui.2015.15.2.6

Aurelia, M. (2005). Padres y desarrollo de los hijos: prácticas de crianza. Estudios Psicológicos, 31(2), 167-177.

Bandura, A. (2003). On the Psychosocial Impact and Mechanisms of Spiritual Modeling. The International Journal for Psychology of Religion, 13(3), 167-173.

Bautista, G., Vera J., Tánori J. \& Valdés, A (2016). Propiedades psicométricas de una escala para medir empatía en estudiantes de secundaria en México. Revista Electrónica Actualidades Investigativas en Educación, 16(3), 1-20. doi: 10.15517/aie. v16i3.25959

Belacchi C. \& Fanina E. (2010). Prosocial/ Hostile roles and emotion comprehension in preschoolers. Aggressive Behavior, 36(1), 371-389.

Betancourt, M. \& Londoño C. (2017). Factores sociodemográficos y psicosociales que diferencian la conducta prosocial y el acoso escolar en jóvenes. Informes 
Psicológicos, 17(1), 159-176. doi: 10.18566/ infpsic.v17n1a09

Blaya, C., Debarbieux, E., Del Rey-Alamillo, R. \& Ortega-Ruiz, R. (2006). Clima y violencia escolar. Un estudio comparativo entre España y Francia. Revista de Educación, 339, 293-315.

Cepeda, E., Pacheco, P. N., García, L. \& Piraquive, C. J. (2008). Acoso escolar a estudiantes de educación básica y media. Revista de Salud Pública, 10(4), 517-528.

Chartier, K., Hesselbrock, M. N. \& Hesselbrock, V. M., (2010). Development and vulnerability factors in adolescent alcohol use. Child and Adolescent Psychiatric Clinics of North America, 19(3), 493-504. doi: 10.1016/j. chc.2010.03.004

Coloroso, B. (2004). The bully, the bullied and the bystander. New York, USA: Harper Colling.

Davis, M. (1980). A multidimensional Approach to Individual Differences in Empathy. JSAS Catalog of Selected Documents in Psychology, 10(85), 2-19.

Da Silva, A., Kodato, S. \& Xavier, F. (2013). Representações sociais de violência em professores da escola pública. Estudos Interdisciplinares em Psicologia, 4(2), 240-257.

Del Rey, R. \& Ortega, R. (2007). Violencia escolar: claves para comprenderla y afrontarla. Escuela abierta, (10), 77-89.

Díaz-Aguado, M.J. (2006). Sexismo, violencia de género y acoso escolar. Propuestas para una prevención integral de la violencia. Revista de Estudios de Juventud, (73), 38-57.
Duarte, K. G. \& Fregoso, D. (2016). Validación de una medida de percepción de los padres sobre factores que se asocian a la violencia escolar (Tesis de pregrado). Universidad de Sonora, México.

Farrington, D. (1993). Understanding and preventing bullying. In M. Tonry (Ed.), Crime and justice, 17 (pp. 381-458). Chicago: The University of Chicago Press.

González, E. N., Peña, M. O. \& Vera, J. A. (2017). Validación de una escala de roles de víctimas y agresores asociados al acoso escolar. Electronic Journal of Research in Educational Psychology, 15(41), 224-239. doi: 10.14204/ejrep.41.16009

Hair, J., Anderson, R., Tatham, R. \& Black, W. (2010). Multivariate data analysis ( $7^{a}$ ed.). Upper Saddle River, NJ: Prentice-Hall.

Hlavaty, K. \& Haselschwerdt, M. L. (2019). Domestic Violence Exposure and Peer Relationships: Exploring the Role of Coercive Control Exposure. Journal of Family Violence, 1-11. doi: 10.1007/ s10896-019-00044-4

Kochenderfer, B.J. \& Ladd, G.W. (1996). Peer victimization: Manifestations and relations to school adjustment in kindergarten. Journal of School Psychology, 34(3), 267-283.

Leff, S., Power, T. \& Goldstein, A. (2004). Outcome measures to assess the effectiveness of bullying prevention programs in the schools. In D. Espelage \& S. Swearer (Eds.), Bullying in American schools: A social-ecological perspective on prevention and intervention (pp. 269-293). Mahwah, NJ: Erlbaum.

López, V., Bilbao, M. \& Rodríguez, J. I. (2012). La sala de clases sí importa: incidencia 
del clima del aula sobre la percepción de intimidación y victimización entre escolares. Universitas Psychologica, 11(1), 91-101.

López L. \& Ovejero, M. (2015). Habilidades comunicativas dentro de la familia: una medida imprescindible contra el acoso escolar en La Rioja. Estudios sobre Educación, 29(4), 123-142. doi: 10.15581/004.29.123-142

Martínez-Ferrer, B., Murgui-Pérez, S., MusituOchoa, G. \& Monreal-Gimeno, M. (2008). El rol del apoyo parental, las actitudes hacia la escuela y la autoestima en la violencia escolar en adolescentes. International Journal of Clinical and Health Psychology, 8(3), 679-692.

Moral, M. \& Ovejero, A. (2014). Relación entre el clima social familiar y las actitudes juveniles ante el acoso escolar. International Journal of Developmental and Educational Psychology. Revista INFAD de Psicología, 5(1), 329-342. doi: 10.17060/ijodaep.2014. n1.v5.690

Muñoz, G. (2008). Violencia escolar en México y en otros países. Comparaciones a partir de los resultados del Instituto Nacional para la Evaluación de la Educación. Revista Mexicana de Investigación Educativa, 13(39), 1195-1228.

Olweus, D. (1993). Bullying at school. Australia: Blackwell Publishing.

Omar, A., Urteaga, A. F., Uribe, H. \& Soares, N. (2010). Capital sociocultural familiar, autoestima u desempeño académico en adolescentes. Revista Latinoamericana de Estudios Educativos (México), 40(2), 93-114.
Parke, R. \& Slaby, R. (1983). The development of aggression. In P. Mussen \& $\mathrm{E}$. Hetherington (Eds.), Handbook of Child Psychology: Socialization, Personality, and Social Development, 4. New York: Wiley.

Pichardo, M. C., Fernández, E. \& Amezcua, J. A. (2002). Importancia del clima social familiar en la adaptación personal y social de los adolescentes. Revista de Psicologia General y Aplicada, 55(4), 575-589.

Rigby, K. (2000). Effects of peer victimization in schools and perceived social support on adolescent well-being. Journal of Adolescence, 23(1), 57-68. doi: 10.1006/ jado.1999.0289

Sanabría, A. \& Uribe, A. (2010). Factores psicosociales de riesgo asociados a conductas problemáticas en jóvenes infractores y no infractores. Diversitas: Perspectivas en Psicología, 6(2), 257-274.

Sharp, S., Thompson, D. \& Arora, T. (2000) How long before it hurts?: An investigation into long term bullying. School PSYchology International, 21(1), 37-46.

Sociedad Mexicana de Psicología. (2007). Código ético del psicólogo (4ª ed.). México: Trillas.

Solís, F. \& Aguiar, R. (2017). Análisis del papel del involucramiento de la familia en la escuela secundaria y su repercusión en el rendimiento académico. Sinéctica, (49), 1-22. Recuperado el 08 de mayo de 2019, de http://www.scielo.org.mx/scielo. php?script=sci_arttext\&pid=S1665-109X2 017000200013\&lng=es\&tlng=es

Spreng, R., McKinnon, M., Mar, R. \& Levine, B. (2009). The Toronto empathy questionnaire: Scale development and 
initial validation of a factor-analytic solution to multiple empathy measures. Journal of Personality Assessment, 91(1), 62-71. doi: 10.1080/00223890802484381

Troop-Gordon, W., Rudolph, K., Sugimura, N. \& Little, T. (2014). Peer victimization in middle childhood impedes adaptive responses to stress: A pathway to depressive symptoms. Journal of Clinical Child \& Adolescent Psychology, 44(3), 432445. doi: 10.1080/15374416.2014.891225

Valdés, A., Carlos, E. \& Arreola, C. (2013). Desarrollo de un instrumento para medir la participación de los padres en la educación escolar de los hijos. REVALUE, 2(1), 1-18.
Valdés, A., Yáñez, A. \& Carlos, E. (2013). Diferencias entre subgrupos de estudiantes involucrados en el bullying: víctimas, agresores-víctimas y agresores. Liberabit, 19(2), 215-222.

Vera, J. A., Fregoso, D. \& Duarte, K. G. (2017). Validación de una escala de control y promoción en habilidades sociales para padres en el contexto de la convivencia escolar. Psicología para América Latina, (28), 100-118. 